\title{
The local-global property for bitangents of plane quartics
}

\author{
Yasuhiro Ishitsuka ${ }^{1,2}$, Tetsushi Ito ${ }^{2,3}$, Tatsuya Ohshita ${ }^{4}$, Takashi Taniguchi ${ }^{5}$ and Yukihiro Uchida ${ }^{6}$ \\ ${ }^{1}$ Center for Science Adventure and Collaborative Research Advancement, Graduate School of \\ Science, Kyoto University, Kitashirakawa Oiwake-cho, Sakyo-ku, Kyoto 606-8502, Japan \\ 2 Department of Mathematics, Faculty of Science, Kyoto University, Kitashirakawa Oiwake-cho, \\ Sakyo-ku, Kyoto 606-8502, Japan \\ ${ }^{3}$ Mathematical Science Team, RIKEN Center for Advanced Intelligence Project (AIP), 1-4-1 \\ Nihonbashi Chuo-ku, Tokyo 103-0027, Japan \\ ${ }^{4}$ Department of Mathematics, Faculty of Science and Technology, Keio University, 3-14-1 \\ Hiyoshi, Kohoku-ku Yokohama, Kanagawa 223-8522, Japan \\ ${ }^{5}$ Department of Mathematics, Graduate School of Science, Kobe University, 1-1 Rokkodai, \\ Nada-ku, Kobe 657-8501, Japan \\ ${ }^{6}$ Department of Mathematical Sciences, Graduate School of Science, Tokyo Metropolitan Uni- \\ versity, 1-1 Minami-Osawa, Hachioji, Tokyo 192-0397, Japan \\ E-mailyasu-ishi@math.kyoto-u.ac.jp
}

Received March 10, 2020, Accepted June 10, 2020

\begin{abstract}
We study the arithmetic of bitangents of smooth quartics over global fields. With the aid of computer algebra systems and using Elsenhans-Jahnel's results on the inverse Galois problem for bitangents, we show that, over any global field of characteristic different from 2 , there exist smooth quartics which have bitangents over every local field, but do not have bitangents over the global field. We give an algorithm to find such quartics explicitly, and give an example over $\mathbb{Q}$. We also discuss a similar problem concerning symmetric determinantal representations.
\end{abstract}

Keywords plane quartic, bitangent, local-global property

Research Activity Group Algorithmic Number Theory and Its Applications

\section{Introduction}

Let $C \subset \mathbb{P}^{2}$ be a smooth quartic over a field $k$ of characteristic different from 2 . It is defined by a homogeneous polynomial $f(X, Y, Z)$ of degree 4 :

$$
C=\left\{[X: Y: Z] \in \mathbb{P}^{2} \mid f(X, Y, Z)=0\right\} .
$$

By Bézout's theorem, the intersection of $C$ with a line $L \subset \mathbb{P}^{2}$ consists of four points, counted with their multiplicities. A line $L \subset \mathbb{P}^{2}$ is called a bitangent of $C$ if one of the following conditions is satisfied:

- $L$ tangents to $C$ at two distinct points, or

- $L$ tangents quadruply to $C$ at one point. (In this case, the line $L$ is also called a hyperflex line of $C$.)

It is known that every smooth quartic has exactly 28 bitangents over an algebraic closure of $k$ [1, Chapter 6].

Bitangents play an important role in the study of the arithmetic of quartics. For example, Bruin-Poonen-Stoll gave an application to the calculation of the MordellWeil group and the rational points [2]. However, since it involves computations on number fields of degree 28 in general, it remains a difficulty to treat the arithmetic of bitangents explicitly.

In this paper, we shall consider the problem whether a given smooth quartic over a global field $K$ has a bitangent over $K$. Concerning this problem, a natural theoretical question is whether the bitangents satisfy the localglobal property (or Hasse principle) or not. The aim of this paper is to answer this question negatively with the aid of computer algebra systems.

Here is the main theorem of this paper.

Theorem 1 Let $K$ be a global field of characteristic different from 2. (It is a finite extension of $\mathbb{Q}$ or $\mathbb{F}_{p}(T)$ with $p \neq 2$.) Then, there exists a smooth quartic $C \subset \mathbb{P}^{2}$ over $K$ such that

- $C$ has a bitangent over $K_{v}$ for every place $v$ of $K$ (including the infinite places when $K$ is a number field), but

- $C$ does not have a bitangent over $K$.

Our proof of Theorem 1 is a combination of certain group theoretic results on $\operatorname{Sp}_{6}\left(\mathbb{F}_{2}\right)$ obtained with the aid of computer algebra systems and the results on the inverse Galois problem for bitangents recently obtained by Elsenhans-Jahnel [3, 4].

Remark 2 After this work was completed, Jahnel and Loughran told the authors that it is also possible to construct smooth quartics satisfying the conditions in Theorem 1 by the results in [5]. (See Remark 12.)

Remark 3 In this paper, we shall also give an algorithm to find quartics failing the local-global property for bitangents explicitly. (See Section 5.) For a given global field $K$, the hardest step is to find a certain Galois extension $L / K$ of degree 32. It seems difficult to give a sharp estimate of the time complexity because this step is related to the effective Chebotarev density theorem. For more details, see Appendix $B$ of the arXiv version (arXiv:2002.10203) of this paper. 


\section{Bitangents and quadratic forms}

Let $k$ be a field of characteristic different from 2, and $k^{\text {sep }}$ a separable closure of $k$. Let $C \subset \mathbb{P}^{2}$ be a smooth quartic over $k$. The Jacobian variety $\operatorname{Jac}(C)$ is an abelian variety of dimension 3 . The group $\operatorname{Jac}(C)[2]$ of $k^{\text {sep }}$ rational points on $\operatorname{Jac}(C)$ killed by 2 is a 6 -dimensional vector space over $\mathbb{F}_{2}$. It is equipped with the action of $\operatorname{Gal}\left(k^{\mathrm{sep}} / k\right)$ and the Weil pairing

$$
\langle,\rangle: \operatorname{Jac}(C)[2] \times \operatorname{Jac}(C)[2] \rightarrow\{ \pm 1\} \cong \mathbb{F}_{2} .
$$

We fix a symplectic basis $\left\{e_{1}, e_{2}, e_{3}, f_{1}, f_{2}, f_{3}\right\}$ of $\operatorname{Jac}(C)[2]$, i.e. $\left\langle e_{i}, e_{j}\right\rangle=\left\langle f_{i}, f_{j}\right\rangle=0$ and $\left\langle e_{i}, f_{j}\right\rangle=\delta_{i, j}$ for all $1 \leq i, j \leq 3$. We have an isomorphism $\operatorname{Jac}(C)[2] \cong$ $\mathbb{F}_{2}^{\oplus 6}$. The action of $\operatorname{Gal}\left(k^{\mathrm{sep}} / k\right)$ gives a continuous map

$$
\rho_{C}: \operatorname{Gal}\left(k^{\mathrm{sep}} / k\right) \rightarrow \operatorname{Sp}(\operatorname{Jac}(C)[2],\langle,\rangle) \cong \operatorname{Sp}_{6}\left(\mathbb{F}_{2}\right) .
$$

A map $Q: \mathbb{F}_{2}^{\oplus 6} \rightarrow \mathbb{F}_{2}$ is called a quadratic form with polar form $\langle$,$\rangle if Q(x+y)-Q(x)-Q(y)=\langle x, y\rangle$ is satisfied for every $x, y \in \mathbb{F}_{2}^{\oplus 6}$. The Arf invariant of $Q$ is defined by

$$
\operatorname{Arf}(Q):=\sum_{1 \leq i \leq 3} Q\left(e_{i}\right) Q\left(f_{i}\right) \in \mathbb{F}_{2},
$$

which is independent of the choice of a symplectic basis.

Let $\Omega^{+}$(resp. $\Omega^{-}$) be the set of quadratic forms with polar form $\langle$,$\rangle whose Arf invariant is 0$ (resp. 1). The set $\Omega^{+}$(resp. $\Omega^{-}$) has 36 (resp. 28) elements. The symplectic group $\operatorname{Sp}_{6}\left(\mathbb{F}_{2}\right)$ acts transitively on $\Omega^{+}$and $\Omega^{-}$.

Lemma 4 (Mumford) There is a bijection between the set $\Omega^{-}$and the set of bitangents of $C$.

See [6] and [7, Proposition 6.2] for details. Since $\operatorname{char}(k) \neq 2$, every bitangent of $C$ is defined over $k^{\text {sep }}$. The bijection in Lemma 4 is equivariant under the action of $\operatorname{Gal}\left(k^{\mathrm{sep}} / k\right)$, where $\operatorname{Gal}\left(k^{\mathrm{sep}} / k\right)$ acts on $\Omega^{-}$through the map $\rho_{C}: \operatorname{Gal}\left(k^{\mathrm{sep}} / k\right) \rightarrow \operatorname{Sp}_{6}\left(\mathbb{F}_{2}\right)$.

Remark 5 For a 'generic' choice of $C$, Harris and Shioda proved that the map $\rho_{C}$ is surjective, at least when $\operatorname{char}(k) \notin\{3,5,7,11,29,1229\}$; see [8, p.721], [9, Theorem 7]. For explicit examples of quartics with surjective $\rho_{C}$, see [9, p.69, Example], [10, p.26, Corollary 3]. Shioda also constructed smooth quartics over $\mathbb{Q}$ such that all the 28 bitangents are defined over $\mathbb{Q}$; see [9, (6.6)].

\section{Group theoretic results}

Let $U_{36} \subset \operatorname{Sp}_{6}\left(\mathbb{F}_{2}\right)$ be the stabilizer of an element of $\Omega^{+}$. The action of $\operatorname{Sp}_{6}\left(\mathbb{F}_{2}\right)$ on $\mathbb{F}_{2}^{\oplus 6} \backslash\{0\}$ is transitive. The stabilizer of a non-zero vector is denoted by $U_{63} \subset \operatorname{Sp}_{6}\left(\mathbb{F}_{2}\right)$. It is known that $U_{36}, U_{63}$ are maximal subgroups of $\operatorname{Sp}_{6}\left(\mathbb{F}_{2}\right)$, and every subgroup of $\operatorname{Sp}_{6}\left(\mathbb{F}_{2}\right)$ of index 36,63 is conjugate to $U_{36}, U_{63}$, respectively.

We shall consider the following condition.

Condition 6 Let $G \subset \operatorname{Sp}_{6}\left(\mathbb{F}_{2}\right)$ be a subgroup. We say $G$ satisfies the condition $(*)^{+}$(resp. $\left.(*)^{-}\right)$if the following conditions are satisfied:

- No element of $\Omega^{+}$(resp. $\left.\Omega^{-}\right)$is fixed by every element of $G$.

- For every $g \in G$, the action of $g$ on $\Omega^{+}$(resp. $\left.\Omega^{-}\right)$ has a fixed point.
The following results can be confirmed by GAP. (A sample source code for GAP can be obtained in Appendix A of the arXiv version (arXiv:2002.10203) of this paper.)

Proposition 7 (1) $U_{36}$ has 296 subgroups, up to conjugation. Among them, 35 subgroups satisfy the condition $(*)^{-}$; all the 35 subgroups are solvable.

(2) $U_{63}$ has 1916 subgroups, up to conjugation. Among them, 548 subgroups satisfy both of the conditions $(*)^{+}$and $(*)^{-} ; 536$ of the 548 subgroups are solvable.

Proposition $8 \mathrm{Sp}_{6}\left(\mathbb{F}_{2}\right)$ has 6 subgroups isomorphic to $\mathbb{F}_{2}^{\oplus 5}$, up to conjugation. All of them satisfy both of the conditions $(*)^{+}$and $(*)^{-}$.

Remark 9 Any subgroup of $U_{36}$ does not satisfy the condition $(*)^{+}$since, by definition, it fixes at least one element of $\Omega^{+}$.

\section{The inverse Galois problems}

The inverse Galois problem asks whether every finite group is realized as the Galois group of a number field over $\mathbb{Q}$. It is an open problem in general. It was proved by Shafarevich for solvable groups. Sonn observed that Shafarevich's solution to the inverse Galois problem yields a Galois extension such that every decomposition group is cyclic; see [11, Theorem 2] for details.

Theorem 10 (Shafarevich, Sonn) Let $G$ be a finite solvable group, and $K$ a global field. Then there exists a finite Galois extension $L / K$ such that $\operatorname{Gal}(L / K)$ is isomorphic to $G$, and that, for every place $v$ of $K$, the decomposition group of $L / K$ at $v$ is cyclic.

Recently, Elsenhans-Jahnel studied an analogue of the inverse Galois problem for bitangents of quartics. Let $k$ be a field of characteristic different from 2, and $\rho: \operatorname{Gal}\left(k^{\mathrm{sep}} / k\right) \rightarrow \operatorname{Sp}_{6}\left(\mathbb{F}_{2}\right)$ a continuous homomorphism. They asked whether $\rho$ is realized as the map $\rho_{C}$ associated with a smooth quartic $C \subset \mathbb{P}^{2}$ over $k$, up to conjugation by an element of $\operatorname{Sp}_{6}\left(\mathbb{F}_{2}\right)$. The following result is proved in [3] (resp. [4]) when the image of $\rho$ is contained in a conjugate of $U_{36}$ (resp. $U_{63}$ ).

Theorem 11 (Elsenhans-Jahnel) If the image of $\rho$ is contained in a conjugate of $U_{36}$ or $U_{63}$, there exists a smooth quartic $C \subset \mathbb{P}^{2}$ over $k$ such that the maps $\rho, \rho_{C}$ are conjugate by an element of $\operatorname{Sp}_{6}\left(\mathbb{F}_{2}\right)$.

Proof of Theorem 1 Theorem 1 is proved by combining Lemma 4, Proposition 7, Theorem 10, and Theorem 11.

(1) Take a solvable subgroup $G \subset \operatorname{Sp}_{6}\left(\mathbb{F}_{2}\right)$ contained in $U_{36}$ or $U_{63}$ which satisfies the condition $(*)^{-}$. Such a subgroup exists by Proposition 7 .

(2) Take a finite Galois extension $L / K$ such that $\operatorname{Gal}(L / K) \cong G$ and, for every place $v$ of $K$, the decomposition group of $L / K$ at $v$ is cyclic. Such an extension exists by Theorem 10 .

(3) Consider the composite of the following maps:

$$
\rho: \operatorname{Gal}\left(K^{\mathrm{sep}} / K\right) \rightarrow \operatorname{Gal}(L / K) \cong G \hookrightarrow \operatorname{Sp}_{6}\left(\mathbb{F}_{2}\right) .
$$


Take a smooth quartic $C \subset \mathbb{P}^{2}$ over $K$ such that the maps $\rho, \rho_{C}$ are conjugate by an element of $\operatorname{Sp}_{6}\left(\mathbb{F}_{2}\right)$. Such a quartic exists by Theorem 11 .

(4) By Lemma 4 , there is a $\operatorname{Gal}\left(K^{\mathrm{sep}} / K\right)$-equivariant bijection between the set $\Omega^{-}$and the set of bitangents of $C$ over $K^{\mathrm{sep}}$. For a place $v$ of $K$, $\operatorname{Gal}\left(K_{v}^{\mathrm{sep}} / K_{v}\right)$ is embedded into $\operatorname{Gal}\left(K^{\mathrm{sep}} / K\right)$. The image $G_{v}:=\rho\left(\operatorname{Gal}\left(K_{v}^{\text {sep }} / K_{v}\right)\right)$ is a cyclic group by (2). Let $g_{v}$ be a generator of $G_{v}$. Since $G$ satisfies the condition $(*)^{-}$by $(1)$, there is an element of $\Omega^{-}$fixed by $g_{v}$. Hence the quartic $C$ has a bitangent over $K_{v}$. Moreover, since no element of $\Omega^{-}$is fixed by every element of $G$, the quartic $C$ does not have a bitangent over $K$. Therefore, the quartic $C$ satisfies all the conditions of Theorem 1 .

(QED)

\section{Description of algorithm}

Here we shall give an algorithm for obtaining smooth quartics failing the local-global property for bitangents.

We take a subgroup $G \subset U_{63} \subset \operatorname{Sp}_{6}\left(\mathbb{F}_{2}\right)$ isomorphic to $\mathbb{F}_{2}^{\oplus 5}$. We can use Proposition 8 for constructing such a subgroup. Explicitly, the group $U_{63}$ is isomorphic to $\left(\mathbb{F}_{2} 2 S_{6}\right) \cap A_{12} ;$ see $[4$, Corollary 2.19$]$. The wreath product $\mathbb{F}_{2} \prec S_{6}$ contains a subgroup isomorphic to $\mathbb{F}_{2}^{\oplus 6}$. Let $G$ be the kernel of the sum $\mathbb{F}_{2}^{\oplus 6} \rightarrow \mathbb{F}_{2}$. Then $G$ is isomorphic to $\mathbb{F}_{2}^{\oplus 5}$, and we have an embedding

$$
G \cong \mathbb{F}_{2}^{\oplus 5} \hookrightarrow\left(\mathbb{F}_{2}<S_{6}\right) \cap A_{12} \cong U_{63} .
$$

Our algorithm consists of 3 steps.

\section{Step 1 (Find a Galois extension $L / K$.)}

Take a Galois extension $L / K$ such that $\operatorname{Gal}(L / K) \cong$ $\mathbb{F}_{2}^{\oplus 5}$ and every decomposition group is cyclic. (The existence of such $L / K$ is guaranteed by Theorem 10.)

Assume that $L$ is described as

$$
L=K\left(\sqrt{a_{1}}, \sqrt{a_{2}}, \sqrt{a_{3}}, \sqrt{a_{4}}, \sqrt{a_{5}}\right)
$$

for some $a_{1}, a_{2}, a_{3}, a_{4}, a_{5} \in K$. We put $a_{6}=$ $a_{1} a_{2} a_{3} a_{4} a_{5} u^{2}$ for some $u \in K^{\times}$and set

$$
F(S, T)=\left(a_{1} S-T\right)\left(a_{2} S-T\right) \ldots\left(a_{6} S-T\right) .
$$

By construction, the splitting field of $F\left(1, T^{2}\right)$ is $L$ and $F(1,0)=\left(a_{1} a_{2} \ldots a_{5} u\right)^{2}$.

Step 2 (Construct a conic bundle $B \subset \mathbb{P}^{1} \times \mathbb{P}^{2}$.)

There exists a unique pair of binary quadratic forms $g(S, T), h(S, T)$ satisfying $\operatorname{det} M(S, T)=-F(S, T)$ and $g(1,0)=a_{1} a_{2} a_{3} a_{4} a_{5} u$, where

$$
M(S, T)=\left(\begin{array}{ccc}
-S T+T^{2} & S T & g(S, T) \\
S T & S^{2} & T^{2} \\
g(S, T) & T^{2} & h(S, T)
\end{array}\right) .
$$

Let $B \subset \mathbb{P}^{1} \times \mathbb{P}^{2}$ be the hypersurface defined by

$$
\left(\begin{array}{lll}
X & Y & Z
\end{array}\right) M(S, T)\left(\begin{array}{l}
X \\
Y \\
Z
\end{array}\right)=0 .
$$

Here $[S: T]$ is the projective coordinate of $\mathbb{P}^{1}$ and $[X$ : $Y: Z]$ is that of $\mathbb{P}^{2}$. The first projection $\mathrm{pr}_{1}: B \rightarrow \mathbb{P}^{1}$ de- fines a conic bundle structure with six degenerate fibers with prescribed Galois action; see [4, Proposition 3.5].

\section{Step 3 (Calculate the branching locus.)}

The composite of the embedding $\iota: B \hookrightarrow \mathbb{P}^{1} \times \mathbb{P}^{2}$ and the projection $\mathrm{pr}_{2}: \mathbb{P}^{1} \times \mathbb{P}^{2} \rightarrow \mathbb{P}^{2}$ is a double cover. Its ramification locus is the desired quartic $C \subset \mathbb{P}^{2}$. (If $C$ is not smooth, then take other parameters $a_{1}, a_{2}, \ldots, a_{5}$ and $u$, and calculate the quartic $C$ again. For a 'generic' choice of $a_{1}, a_{2}, \ldots, a_{5}$ and $u$, the quartic $C$ is smooth; see [4, Proposition 3.5].)

Applying the above algorithm, we can construct a smooth quartic $C \subset \mathbb{P}^{2}$ over $K$ such that the map $\rho_{C}$ is conjugate to the composite of the following maps:

$$
\operatorname{Gal}\left(K^{\mathrm{sep}} / K\right) \rightarrow \operatorname{Gal}(L / K) \cong G \hookrightarrow \operatorname{Sp}_{6}\left(\mathbb{F}_{2}\right) .
$$

The smooth quartic $C \subset \mathbb{P}^{2}$ satisfies all the conditions of Theorem 1 by Proposition 8 .

Remark 12 (Jahnel-Loughran) Here is another construction of smooth quartics satisfying the conditions of Theorem 1 by the results in [5]. Take two closed points $P, Q$ of the projective plane $\mathbb{P}^{2}$ over $K$ (as a $K$-scheme) of degree 3,4, respectively, such that

- the union $P \sqcup Q$ lies in general position, and

- the blow-up of $\mathbb{P}^{2}$ along $Q$ is a del Pezzo surface of degree 5 which does not satisfy the local-global property for lines; see [5, Section 3.6].

Let $X$ be the blow-up of $\mathbb{P}^{2}$ along $P \sqcup Q$. The branching locus $C \subset \mathbb{P}^{2}$ of the anticanonical map $\pi: X \rightarrow \mathbb{P}^{2}$ is a smooth quartic. We have a 2: 1 map from the 56 lines on $X$ to the 28 bitangents of $C$. For every place $v$ of $K, X$ has a line over $K_{v}$ by [5, Lemma 3.9]. Hence $C$ has a bitangent over $K_{v}$. Moreover, $C$ does not have a bitangent over $K$. In fact, if $L \subset \mathbb{P}^{2}$ is a bitangent over $K$, its inverse image $\pi^{-1}(L)=L_{1} \cup L_{2}$ is a union of two lines on $X$. The lines on $X$ are classified into the types (i)-(iv) in [4, Remark 2.8]. Since $L_{1}, L_{2}$ have different types and the type of a line is Galois invariant, both of $L_{1}, L_{2}$ are defined over $K$. It contradicts [5, Lemma 3.8].

\section{An example}

We consider the case $K=\mathbb{Q}$. We put $b_{1}=-1, b_{2}=$ $17, b_{3}=89, b_{4}=257, b_{5}=769$, and put $K_{i}=\mathbb{Q}\left(\sqrt{b_{i}}\right)$ for every $1 \leq i \leq 5$. Then, for every $1 \leq i \leq 5$, only one prime number is ramified in $K_{i} / \mathbb{Q}$. For every $1 \leq i, j \leq 5$ with $i \neq j$, the prime number $p_{i}$ splits in $K_{j} / \mathbb{Q}$. (Here $p_{i}$ is the unique prime number ramified in $K_{i} / \mathbb{Q}$.) We put $L=K_{1} K_{2} K_{3} K_{4} K_{5}$, and $a_{1}=b_{1} b_{5}, a_{2}=b_{2} b_{4}, a_{3}=b_{3}$, $a_{4}=b_{4}, a_{5}=b_{5}, u=-b_{4}^{-1} b_{5}^{-1}$.

Then $L / K$ satisfies the conditions in Step 1. We have

$$
\begin{aligned}
& F(S, T)=(-769 S-T)(4369 S-T)(89 S-T) \\
&(257 S-T)(769 S-T)(-1513 S-T), \\
& g(S, T)=\frac{1}{8}\left(2392149832 S^{2}+35008837 S T+12804 T^{2}\right), \\
& h(S, T)=-\frac{1}{64}\left(251582881045706064 S^{2}\right. \\
&\left.+1084638148302617 S T+594847875240 T^{2}\right) .
\end{aligned}
$$


The quartic is computed as

$$
\begin{aligned}
& 4096 X^{4}-16384 X^{3} Y-9869943810048 X^{3} Z \\
& \quad+143396196352 X^{2} Y Z-52445184 X Y^{2} Z \\
& \quad-32768 Y^{3} Z+64826445425191482752 X^{2} Z^{2} \\
& \quad-277686962456893696 X Y Z^{2} \\
& \quad+152281056061440 Y^{2} Z^{2} \\
& \quad-917870567374331469445024 X Z^{3} \\
& +128810435095401504768 Y Z^{3} \\
& +
\end{aligned}
$$

It gives an example of smooth quartics over $\mathbb{Q}$ failing the local-global property for bitangents.

\section{The local-global property for symmet- ric determinantal representations}

We say a smooth quartic $C \subset \mathbb{P}^{2}$ over a field $k$ admits a symmetric determinantal representation over $k$ if there exist symmetric matrices $M_{1}, M_{2}, M_{3} \in \operatorname{Mat}_{4}(k)$ of size $4 \times 4$ such that the equation

$$
\operatorname{det}\left(X M_{1}+Y M_{2}+Z M_{3}\right)=0
$$

defines the quartic $C \subset \mathbb{P}^{2}$. (For explicit examples, see [12].)

By the same method as above, it is possible to obtain smooth quartics failing the local-global property for symmetric determinantal representations.

Theorem 13 For any global field $K$ of characteristic different from 2 , there exists a smooth quartic $C \subset \mathbb{P}^{2}$ over $K$ such that

- $C$ admits a symmetric determinantal representation over $K_{v}$ for every place $v$ of $K$, but

- $C$ does not admit a symmetric determinantal representation over $K$.

Here is a sketch of the proof. For a smooth quartic $C \subset \mathbb{P}^{2}$ with a $K$-rational point, it admits a symmetric determinantal representation over $K$ if and only if there exists a $\operatorname{Gal}\left(K^{\mathrm{sep}} / K\right)$-invariant quadratic form in $\Omega^{+}$; see [7, Theorem 2.2, Corollary 6.3]. Taking a subgroup $G \subset U_{63}$ satisfying the condition $(*)^{+}$, we find smooth quartics over $K$ satisfying the conditions of Theorem 13 by the same way as in the case of bitangents.

Example 14 Quartics constructed by the algorithm in Section 5 always have the $\mathbb{Q}$-rational point $[0: 1: 0]$. By Proposition 8, the example of quartic in Section 6 satisfies the conditions of Theorem 13. (But the quartics constructed by the method described in Remark 12 do not satisfy these conditions.)

Remark 15 In [7], it is proved that smooth quartics over number fields do not satisfy the local-global property for symmetric determinantal representations. The quartics constructed in [7] are defined over number fields of large degree. For quartics over $\mathbb{Q}$, this problem was stated in [7, Problem 1.6 (1)], but not answered there.

Remark 16 Theorem 13 does not hold in characteristic 2. In fact, over a global field of characteristic 2, any smooth plane curve of any degree satisfies the localglobal property for symmetric determinantal representations; see [13] for details.

\section{Acknowledgments}

The authors would like to thank Jörg Jahnel and Daniel Loughran for explaining how to construct quartics failing the local-global property for bitangents by the results in [5]; see Remark 12. The authors would like to thank the referee for comments and constructive advice. Y. I.'s work was supported by JSPS KAKENHI Grant Number 16K17572. T. I.'s work was supported by JSPS KAKENHI Grant Number 20674001 and 26800013. T. O.'s work was supported by JSPS KAKENHI Grant Number 26800011 and 18H05233. T. T.'s work was supported by JSPS KAKENHI Grant Number 17H02835. Y. U.'s work was supported by JSPS KAKENHI Grant Number 20K03517. This work was supported by the Sumitomo Foundation FY2018 Grant for Basic Science Research Projects (Grant Number 180044). Most of calculations were done with the aid of GAP [14], Maxima [15], Sage [16].

\section{References}

[1] I. Dolgachev, Classical Algebraic Geometry: A Modern View, Cambridge University Press, Cambridge, 2012.

[2] N. Bruin, B. Poonen and M. Stoll, Generalized explicit descent and its application to curves of genus 3, Forum Math. Sigma, 4 (2016), e6, 80 pages.

[3] A-S. Elsenhans and J. Jahnel, On plane quartics with a Galois invariant Cayley octad, Eur. J. Math., 5 (2019), 1156-1172.

[4] A-S. Elsenhans and J. Jahnel, Plane quartics with a Galoisinvariant Steiner hexad, Int. J. Number Theory, 15 (2019), 1075-1109.

[5] J. Jahnel and D. Loughran, The Hasse principle for lines on del Pezzo surfaces, Int. Math. Res. Not., 2015 (2015), 12877 12919.

[6] D. Mumford, Theta characteristics of an algebraic curve, Ann. Sci. Éc. Norm. Supér. (4), 4 (1971), 181-192.

[7] Y. Ishitsuka and T. Ito, The local-global principle for symmetric determinantal representations of smooth plane curves, Ramanujan J., 43 (2017), 141-162.

[8] J. Harris, Galois groups of enumerative problems, Duke Math. J., 46 (1979), 685-724.

[9] T. Shioda, Plane quartics and Mordell-Weil lattices of type $E_{7}$, Comment. Math. Univ. St. Pauli, 42 (1993), 61-79.

[10] R. Erné, Construction of a del Pezzo surface with maximal Galois action on its Picard group, J. Pure Appl. Algebra, 97 (1994), 15-27.

[11] J. Sonn, Polynomials with roots in $\mathbb{Q}_{p}$ for all $p$, Proc. Amer. Math. Soc., 136 (2008), 1955-1960.

[12] Y. Ishitsuka, T. Ito and T. Ohshita, On algorithms to obtain linear determinantal representations of smooth plane curves of higher degree, JSIAM Letters, 11 (2019), 9-12.

[13] Y. Ishitsuka and T. Ito, The local-global principle for symmetric determinantal representations of smooth plane curves in characteristic two, J. Pure Appl. Algebra, 221 (2017), 1316-1321.

[14] The GAP Group, GAP - Groups, Algorithms, and Programming, Version 4.10.2, 2019, https://www.gap-system.org.

[15] Maxima.sourceforge.net. Maxima, a Computer Algebra System (Version 5.41.0), 2017, http://maxima.sourceforge. net.

[16] SageMath, the Sage Mathematics Software System (Version 8.9), The Sage Developers, 2019, http://www.sagemath.org. 